\title{
Externally driven one-dimensional Ising model
}

\author{
Amir Aghamohammadi ${ }^{a}$ 1, Cina Aghamohammadi ${ }^{b}$ 2, \& Mohammad Khorrami ${ }^{a}{ }^{3}$ \\ ${ }^{a}$ Department of Physics, Alzahra University, Tehran 19938-91176, Iran \\ ${ }^{b}$ Department of Electrical Engineering, Sharif University of Technology, 11365- \\ 11155, Tehran, Iran
}

\begin{abstract}
A one dimensional kinetic Ising model at a finite temperature on a semiinfinite lattice with time varying boundary spins is considered. Exact expressions for the expectation values of the spin at each site are obtained, in terms of the time dependent boundary condition and the initial conditions. The solution consists of a transient part which is due to the initial condition, and a part driven by the boundary. The latter is an evanescent wave when the boundary spin is oscillating harmonically. Low- and high-frequency limits are investigated with greater detail. The total magnetization of the lattice is also obtained. It is seen that for any arbitrary rapidly varying boundary conditions, this total magnetization is equal to the the boundary spin itself, plus essentially the time integral of the boundary spin. A nonuniform model is also investigated.
\end{abstract}

\footnotetext{
${ }^{1}$ e-mail: mohamadi@alzahra.ac.ir

${ }^{2}$ e-mail: c_aghamohammadi@yahoo.com

3 e-mail: mamwad@mailaps.org
} 


\section{Introduction}

Dynamical spin systems have played a central role in non-equilibrium statistical models. The Ising model is widely studied in statistical mechanics, as it is simple and allows one to understand many features of phase transitions. The non-equilibrium properties of the Ising model follow from the spin dynamics. In his article 1, Glauber introduced a dynamical model formulating the dynamics of spins, based on the rates coming from a detailed balance analysis. It is a simple non-equilibrium model of interacting spins with spin flip dynamics. An extension of the kinetic Ising model with nonuniform coupling constants on a one-dimensional lattice was introduced in [2]. In [3], a damage spreading method was used to study the sensitivity of the time evolution of a kinetic Ising model with Glauber dynamics against the initial conditions. The full time dependence of the space-dependent magnetization and of the equal time spin-spin correlation functions were studied in [4. Non-equilibrium two-time correlation and response functions for the ferromagnetic Ising chain with Glauber dynamics have been studied in [5, 6]. The dynamics of a left-right asymmetric Ising chain has been studied in [7]. The response function to an infinitesimal magnetic field for the Ising-Glauber model with arbitrary exchange couplings was addressed in 8. In [9], a Glauber model on a one-dimensional lattice with boundaries was studied, for both ferromagnetic and anti-ferromagnetic couplings. The largetime behavior of the one-point function was studied. It was shown that the system exhibits a dynamical phase transition, which is controlled by the rate of spin flip at the boundaries.

It was shown in [10, 11] that for a nonuniform extension of the kinetic Ising model, there are cases where the system exhibits static and dynamical phase transitions. Using a transfer matrix method, it was shown that there are cases where the system exhibits a static phase transition, which is a change of behavior of the static profile of the expectation values of the spins near end points 10. Using the same method, it was shown in [1] that a dynamic phase transition could occur as well: there is a fast phase where the relaxation time is independent of the reaction rates at the boundaries, and a slow phase where the relaxation time does depend on the reaction rates at the boundaries.

Most of the studies on reaction diffusion models have been on cases where the boundary conditions are constant in time. Among the few models with time dependent boundary conditions, is the asymmetric simple exclusion process on a semi-infinite chain coupled at the end to a reservoir with a particle density that changes periodically in time [12. The situation is similar regarding the case of the kinetic Ising model as well. Among the exceptions are the study of the dynamical response of a two-dimensional Ising model subject to a square-wave external field [13, and the study of a harmonic oscillator linearly coupled with a linear chain of Ising spins [14, 15].

In this article a one dimensional Ising model at temperature $T$ on a semiinfinite lattice with time varying boundary spin is investigated. The paper is organized as follows. In section 2 a brief review of the formalism is presented, mainly to introduce the notation. In section 3 , a semi-infinite lattice with 
oscillating boundary spin is studied. The exact solution for the expectation values of the spin at any site is obtained. It is shown that there the boundary produces an evanescent wave in the lattice. The low and high frequency limits are studied in greater detail. The total magnetization of the lattice, $M(t)$, is also obtained. It is shown that for rapidly changing boundary conditions, the total magnetization is equal to the the boundary spin itself, plus something proportional to the time integral of the boundary spin. A nonuniform model in also investigated. It is shown that its evolution operator eigenvalues are real. For the specific case of a two-part lattice with each part being homogeneous, the reflection and transmission coefficients corresponding to a harmonic source at the end of the lattice are calculated. Finally, section 4 is devoted to the concluding remarks.

\section{One-dimensional Ising model with nonuniform coupling constants}

Consider an Ising model on a one-dimensional lattice with $L$ sites, labeled from 1 to $L$. At each site of the lattice there is a spin interacting with its nearest neighboring sites according to the Ising Hamiltonian. At the boundaries there are fixed magnetic fields. Denoting the spin at the site $j$ by $s_{j}$, and the magnetic field at the sites 1 and $L$ by $\mathfrak{B}_{1}$ and $\mathfrak{B}_{L}$, one has for the Ising Hamiltonian

$$
\mathcal{H}=-\sum_{\alpha=1+\mu}^{L-\mu} J_{\alpha} s_{\alpha-\mu} s_{\alpha+\mu}-\mathfrak{B}_{1} s_{1}-\mathfrak{B}_{L} s_{L} .
$$

where $J_{\alpha}$ is the coupling constant in the link $\alpha$, and

$$
\mu=\frac{1}{2} .
$$

The link $\alpha$ links the sites $\alpha-\mu$ and $\alpha+\mu$, so that $\alpha \pm \mu$ are integers, and $\alpha$ runs from $\mu$ up to $(L-\mu)$. Throughout this paper, sites are denoted by Latin letters which represent integers, while links are denoted by Greek letters which represent integers plus one half $(\mu)$. The spin variable $s_{j}$ takes the values +1 for spin up $(\uparrow)$, or -1 for spin down $(\downarrow)$. Define

$$
K_{\alpha}:= \begin{cases}\beta J_{\alpha}, & 1<\alpha<L \\ \beta \mathfrak{B}_{1}, & \alpha=\mu \\ \beta \mathfrak{B}_{L}, & \alpha=L+\mu\end{cases}
$$

where

$$
\beta:=\frac{1}{k_{\mathrm{B}} T},
$$

and $k_{\mathrm{B}}$ is the Boltzmann's constant, and $T$ is the temperature. Denoting the reaction rate from the configuration $A$ to the configuration $B$ by $\omega(A \rightarrow B)$, 
and assuming that in each step only one spin flips, detailed balance demands the following for the reaction rates.

$$
\begin{aligned}
\omega\left[\left(S^{\prime}, s_{j}\right) \rightarrow\left(S^{\prime},-s_{j}\right)\right]= & \Gamma_{j}\left[1-s_{j} \tanh \left(K_{j-\mu} s_{j-1}+K_{j+\mu} s_{j+1}\right)\right], \\
& 1<j<L, \\
\omega\left[\left(S^{\prime}, s_{1}\right) \rightarrow\left(S^{\prime},-s_{1}\right)\right]= & \Gamma_{1}\left[1-s_{1} \tanh \left(K_{\mu}+K_{1+\mu} s_{2}\right)\right], \\
\omega\left[\left(S^{\prime}, s_{L}\right) \rightarrow\left(S^{\prime},-s_{L}\right)\right]= & \Gamma_{L}\left[1-s_{L} \tanh \left(K_{L-\mu} s_{L-1}+K_{L+\mu}\right)\right] .
\end{aligned}
$$

$\Gamma_{j}$ 's are independent of the configurations. For simplicity, we take them to be independent of the site. Then, rescaling the time they are set equal to one. is

So the evolution equation for the expectation value of the spin in the site $j$

$$
\begin{aligned}
\frac{\mathrm{d}}{\mathrm{d} t}\left\langle s_{j}\right\rangle= & -2\left\langle s_{j}\right\rangle+\left[\tanh \left(K_{j-\mu}+K_{j+\mu}\right)+\tanh \left(K_{j-\mu}-K_{j+\mu}\right)\right]\left\langle s_{j-1}\right\rangle \\
& +\left[\tanh \left(K_{j-\mu}+K_{j+\mu}\right)-\tanh \left(K_{j-\mu}-K_{j+\mu}\right)\right]\left\langle s_{j+1}\right\rangle, \quad 1<j<L \\
\frac{\mathrm{d}}{\mathrm{d} t}\left\langle s_{1}\right\rangle= & -2\left\langle s_{1}\right\rangle+\left[\tanh \left(K_{\mu}+K_{1+\mu}\right)+\tanh \left(K_{\mu}-K_{1+\mu}\right)\right] \\
& +\left[\tanh \left(K_{\mu}+K_{1+\mu}\right)-\tanh \left(K_{\mu}-K_{1+\mu}\right)\right]\left\langle s_{2}\right\rangle \\
\frac{\mathrm{d}}{\mathrm{d} t}\left\langle s_{L}\right\rangle= & -2\left\langle s_{L}\right\rangle+\left[\tanh \left(K_{L-\mu}+K_{L+\mu}\right)+\tanh \left(K_{L-\mu}-K_{L+\mu}\right)\right]\left\langle s_{L-1}\right\rangle \\
& +\left[\tanh \left(K_{L-\mu}+K_{L+\mu}\right)-\tanh \left(K_{L-\mu}-K_{L+\mu}\right)\right] .
\end{aligned}
$$

These can be written in the form

$$
\begin{aligned}
\frac{\mathrm{d}}{\mathrm{d} t}\left\langle s_{j}\right\rangle= & -2\left\langle s_{j}\right\rangle+\left[\tanh \left(K_{j-\mu}+K_{j+\mu}\right)+\tanh \left(K_{j-\mu}-K_{j+\mu}\right)\right]\left\langle s_{j-1}\right\rangle \\
& +\left[\tanh \left(K_{j-\mu}+K_{j+\mu}\right)-\tanh \left(K_{j-\mu}-K_{j+\mu}\right)\right]\left\langle s_{j+1}\right\rangle, \quad 1 \leq j \leq L \\
\left\langle s_{0}\right\rangle= & 1 \\
\left\langle s_{L+1}\right\rangle= & 1 .
\end{aligned}
$$

\section{Time varying boundary conditions on a semi- infinite lattice}

Consider a lattice for which the boundary spins $\left(s_{0}\right.$ and $\left.s_{L+1}\right)$ are externally controlled, but the reactions at the internal sites satisfy detailed balance. The evolution equation is then the same as (9), but combined with boundary conditions different from (10) and (11). A semi-infinite lattice the boundary of which is externally controlled, is obtained by letting $L$ tend to infinity, and using the following boundary conditions

$$
\begin{aligned}
& \left\langle s_{0}\right\rangle=f(t), \\
& \left\langle s_{j}\right\rangle \text { does not blow up as } j \text { tends to infinity, }
\end{aligned}
$$


instead of (10) and (11).

A general solution of (9), combined with (12) and (13), can be written as the sum of a particular solution plus a general solution of (9), combined with the homogeneous boundary conditions.

\subsection{Semi-infinite lattice with uniform couplings: the ho- mogeneous solution}

For a lattice with uniform couplings, $K_{\alpha}$ 's are denoted by $K$. The solution to the homogenous equation (vanishing $f$ ) is denoted by $\left\langle s_{j}\right\rangle_{\mathrm{h}}$. One arrives at

$$
\frac{\mathrm{d}}{\mathrm{d} t}\left\langle s_{j}\right\rangle_{\mathrm{h}}=-2\left\langle s_{j}\right\rangle_{\mathrm{h}}+[\tanh (2 K)]\left(\left\langle s_{j-1}\right\rangle_{\mathrm{h}}+\left\langle s_{j+1}\right\rangle_{\mathrm{h}}\right), \quad 0<j .
$$

Defining

$$
\left\langle s_{j}\right\rangle_{\mathrm{h}}:=-\left\langle s_{-j}\right\rangle_{\mathrm{h}}, \quad j<0,
$$

one arrives at

$$
\frac{\mathrm{d}}{\mathrm{d} t}\left\langle s_{j}\right\rangle_{\mathrm{h}}=-2\left\langle s_{j}\right\rangle_{\mathrm{h}}+[\tanh (2 K)]\left(\left\langle s_{j-1}\right\rangle_{\mathrm{h}}+\left\langle s_{j+1}\right\rangle_{\mathrm{h}}\right),
$$

which holds for all integers $j$. Denoting the linear operator acting on $\left\langle s_{l}\right\rangle$ 's in the right-hand side of (16) by $h$, the above equation is of the form

$$
\frac{\mathrm{d}}{\mathrm{d} t}\left\langle s_{j}\right\rangle_{\mathrm{h}}=h_{j}^{l}\left\langle s_{l}\right\rangle_{\mathrm{h}}
$$

where $h_{j}^{l}$ 's are the matrix elements of $h$. Defining the generating function $G$ through

$$
G(z, t):=\sum_{j=-\infty}^{\infty} z^{j}\left\langle s_{j}\right\rangle_{\mathrm{h}}(t)
$$

one arrives at

$$
\frac{\partial G}{\partial t}=\left[-2+\left(z+z^{-1}\right) \tanh (2 K)\right] G,
$$

resulting in

$$
\begin{aligned}
G(z, t) & =\exp \left\{\left[-2+\left(z+z^{-1}\right) \tanh (2 K)\right] t\right\} G(z, 0) \\
& =\exp (-2 t) \sum_{k=-\infty}^{\infty} z^{k} \mathrm{I}_{k}[2 t \tanh (2 K)] G(z, 0), \\
& =\exp (-2 t) \sum_{j=-\infty}^{\infty} z^{j} \sum_{l=-\infty}^{\infty} \mathrm{I}_{j-l}[2 t \tanh (2 K)]\left\langle s_{l}\right\rangle_{\mathrm{h}}(0),
\end{aligned}
$$


where $\mathrm{I}_{k}$ is the modified Bessel function of first kind of order $k$. (20) results in

$$
\begin{aligned}
\left\langle s_{j}\right\rangle_{\mathrm{h}}(t) & =\exp (-2 t) \sum_{l=-\infty}^{\infty} \mathrm{I}_{j-l}[2 t \tanh (2 K)]\left\langle s_{l}\right\rangle_{\mathrm{h}}(0), \\
& =\exp (-2 t) \sum_{l=1}^{\infty}\left\{\mathrm{I}_{j-l}[2 t \tanh (2 K)]-\mathrm{I}_{j+l}[2 t \tanh (2 K)]\right\}\left\langle s_{l}\right\rangle_{\mathrm{h}}(0) .
\end{aligned}
$$

Using the large argument behavior of the modified Bessel functions, it is seen that

$$
\left\langle s_{j}\right\rangle_{\mathrm{h}}(t) \sim \exp \{-2[1-\tanh (2 K)] t\}, \quad j>0,
$$

showing that the homogeneous solution tends to zero at large times.

\subsection{Semi-infinite lattice with uniform couplings: the par- ticular solution corresponding to harmonic boundary conditions}

The harmonic boundary condition is

$$
\left\langle s_{0}\right\rangle=\operatorname{Re}\left[\sigma_{0} \exp (-\mathrm{i} \omega t)\right]
$$

The following ansatz for a particular solution $\left\langle s_{j}\right\rangle_{\mathrm{p}}$ to equations (9) and (12)

$$
\left\langle s_{j}\right\rangle_{\mathrm{p}}=\operatorname{Re}\left[\sigma_{j} \exp (-\mathrm{i} \omega t)\right]
$$

results in

$$
(\mathrm{i} \omega-2) \sigma_{j}+[\tanh (2 K)]\left(\sigma_{j+1}+\sigma_{j-1}\right)=0 .
$$

This has a solution of the form

$$
\sigma_{j}=c z^{j},
$$

where $z$ satisfies

$$
z+z^{-1}=\frac{-i \omega+2}{\tanh (2 K)} .
$$

It is obvious that changing the sign of $K$ results in changing the sign of $z$, while changing the sign of $\omega$ results in changing $z$ to its complex conjugate. So it is sufficient to consider only nonegative values of $K$ and $\omega$. From now on, it is assumed that $K$ and $\omega$ are nonnegative. (27) has two solution for $z$, which are inverse of each other, and none are unimodular. The boundary condition at infinity imposes that of the two solutions of type (26), only that solution is acceptable which corresponds to the root of (27) with modulus less than one. From now, only this root is denoted by $z$ :

$$
z:=r \exp (\mathrm{i} \theta)
$$


where $r$ and $\theta$ are real and $r$ is positive and less that one. The solution to (25) is then

$$
\sigma_{j}=\sigma_{0} z^{j} .
$$

As $|z|$ is less than one, the particular solution (24) describes an evanescent wave. Obviously, the rate of decay length and the phase speed, $\ell$ and $v$ respectively, satisfy

$$
\begin{aligned}
& \ell=-\frac{1}{\ln r}, \\
& v=\frac{\theta}{\omega} .
\end{aligned}
$$

As the homogenous solution (21) tends to zero for large times, the particular solution (24) is in fact the large times solution to the problem of harmonic boundary condition.

Defining

$$
\begin{aligned}
a & :=\frac{\omega}{2}, \\
b & :=\tanh 2 K, \\
u & :=\frac{r+r^{-1}}{2},
\end{aligned}
$$

the real and imaginary parts of (27) read

$$
\begin{aligned}
u \cos \theta & =\frac{1}{b}, \\
\sqrt{u^{2}-1} \sin \theta & =\frac{a}{b} .
\end{aligned}
$$

So $u$ satisfies

$$
b^{2} u^{4}-\left(a^{2}+b^{2}+1\right) u^{2}+1=0,
$$

from which one arrives, for the solution which is larger than one, at

$$
u=\left(\frac{1+a^{2}+b^{2}+\sqrt{\left(1+a^{2}+b^{2}\right)^{2}-4 b^{2}}}{2 b^{2}}\right)^{1 / 2} .
$$

This is increasing with respect to $a$, and decreasing with respect to $b$. Noting that

$$
\frac{\mathrm{d} u}{\mathrm{~d} r}=\frac{1}{2}\left(1-\frac{1}{r^{2}}\right),
$$

which shows that $u$ is decreasing with respect to $r$, it is seen that $r$ is decreasing with respect to $\omega$, and increasing with respect to $K$. One also has

$$
r=u-\sqrt{u^{2}-1} .
$$


Regarding $\theta$, differentiating the first equation in (32) with respect to $a$, one has

$$
\cos \theta \frac{\partial u}{\partial a}-u \sin \theta \frac{\partial \theta}{\partial a}=0,
$$

resulting in

$$
\begin{aligned}
\frac{\partial \theta}{\partial a} & =\frac{\cos \theta}{u \sin \theta} \frac{\partial u}{\partial a}, \\
& =\frac{b^{2} u^{2}-b^{2}}{b u \sqrt{\left(1+a^{2}+b^{2}\right)^{2}-4 b^{2}}} \frac{\sin \theta}{a}, \\
& =\frac{b^{2} u^{2}-b^{2}}{b u\left(b^{2} u^{2}-u^{-2}\right)} \frac{\sin \theta}{a} .
\end{aligned}
$$

Equation (34) shows that

$$
b u \geq 1
$$

from which it is seen that

$$
0<\frac{\partial \theta}{\partial a} \leq \frac{\sin \theta}{a} .
$$

The first inequality shows $\theta$ is an increasing function of $a$, so it is an increasing function of $\omega$. The second inequality results in

$$
\frac{\partial \theta}{\partial a} \leq \frac{\theta}{a},
$$

which shows that $(\theta / a)$ is a decreasing function of $a$. So $(a / \theta)$ is an increasing function of $a$, or $(\omega / \theta)$ is an increasing function of $\omega$.

One also has

$$
\frac{\partial\left(2 b^{2} u^{2}\right)}{\partial b^{2}}=1+\frac{a^{2}+b^{2}-1}{\sqrt{\left(1+a^{2}+b^{2}\right)^{2}-4 b^{2}}},
$$

and as

$$
\sqrt{\left(1+a^{2}+b^{2}\right)^{2}-4 b^{2}} \geq 1-b^{2},
$$

it turns out that $(b u)$ is increasing with $b$, so that $\theta$ is increasing with $b$. Hence $(\omega / \theta)$ is decreasing with $K$.

The asymptotic behavior of $r$ and $\theta$ is summarized as

$$
r=\left\{\begin{array}{ll}
\frac{\tanh K,}{\tanh (2 K)} & \omega \ll 1, \\
\frac{\tanh (2 K)}{\sqrt{4+\omega^{2}},} & 1 \ll \omega, \\
\frac{\sqrt{8+\omega^{2}+\omega \sqrt{16+\omega^{2}}}-\sqrt{\omega^{2}+\omega \sqrt{16+\omega^{2}}},}{\sqrt{8}}, & 1 \ll K
\end{array},\right.
$$




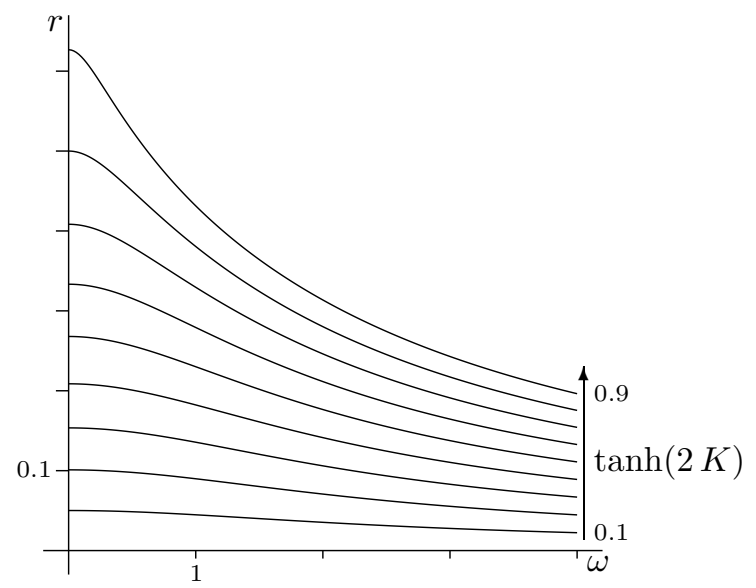

Figure 1:

The plot of $r$ versus $\omega$ for different values of $\tanh (2 K)$

and

$$
\theta= \begin{cases}\frac{\omega \cosh (2 K)}{2}, & \omega \ll 1, \\ \frac{\pi}{2}, & 1 \ll \omega, \\ \tan ^{-1} \frac{\omega}{2}, & K \ll 1, \\ \cos ^{-1} \sqrt{\frac{8+\omega^{2}-\omega \sqrt{16+\omega^{2}}}{8}}, & 1 \ll K\end{cases}
$$

Among other things, it is seen that the phase speed, at low frequencies approaches the constant value $2 /[\cosh (2 K)]$, while at high frequencies varies like $(2 \omega / \pi)$.

Figure 1 is a plot of $r$ versus $\omega$ for different values of $\tanh (2 K)$ from 0.1 to 0.9 . Figure 2 is a plot of the phase speed $(\omega / \theta)$ versus $\omega$ for different values of $\tanh (2 \mathrm{~K})$ from 0.1 to 0.9 .

The total magnetization, defined as the sum of the expectation values of the spins, is denoted by $M$. At large times only the particular solution contributes to the magnetization. So,

$$
M=\operatorname{Re}\left[\frac{\sigma_{0}}{1-z} \exp (-\mathrm{i} \omega t)\right], \quad t \rightarrow \infty .
$$

For the time-independent boundary condition, this leads to

$$
M=\frac{\sigma_{0}}{1-\tanh K}, \quad(t \rightarrow \infty, \omega=0) .
$$




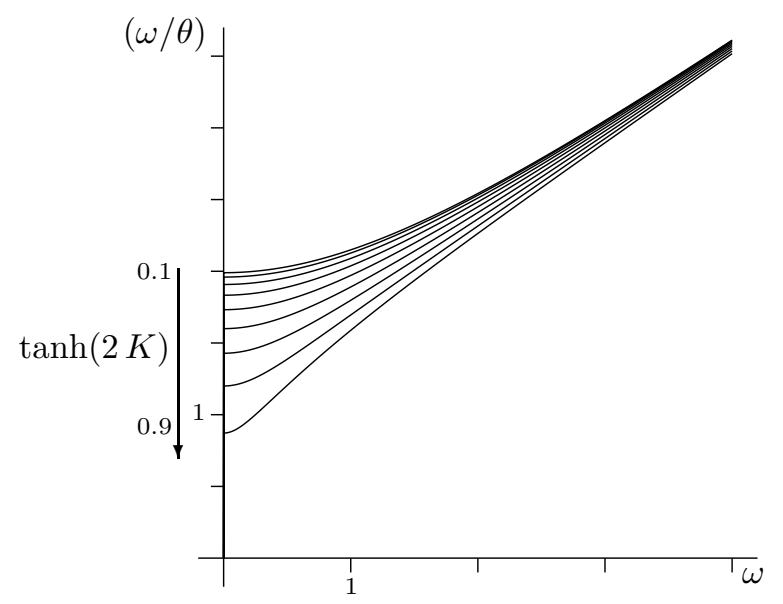

Figure 2:

The plot of the phase speed $(\omega / \theta)$ versus $\omega$ for different values of $\tanh (2 K)$

For high frequencies,

$$
M=\operatorname{Re}\left\{\sigma_{0}\left[1-\frac{\tanh (2 K)}{-\mathrm{i} \omega}\right]^{-1} \exp (-\mathrm{i} \omega t)\right\}, \quad(t \rightarrow \infty, \omega \rightarrow \infty) .
$$

This can be simplified to

$$
\begin{aligned}
M & =\operatorname{Re}\left\{\sigma_{0}\left[1+\frac{\tanh (2 K)}{-\mathrm{i} \omega}\right] \exp (-\mathrm{i} \omega t)\right\}, \\
& =\left\langle s_{0}\right\rangle(t)+[\tanh (2 K)]\left[S_{0}(t)-\bar{S}_{0}\right], \quad(t \rightarrow \infty, \omega \rightarrow \infty),
\end{aligned}
$$

where

$$
\begin{aligned}
S_{0}(t) & :=\int_{0}^{t} \mathrm{~d} t^{\prime}\left\langle s_{0}\right\rangle\left(t^{\prime}\right), \\
\bar{S}_{0} & :=\lim _{T \rightarrow \infty}\left[\frac{1}{T} \int_{T} \mathrm{~d} t S_{0}(t)\right] .
\end{aligned}
$$

One then arrives at a similar result for the magnetization when the boundary condition is any arbitrary rapidly varying function of time (so that its low frequency components are negligible):

$$
\begin{aligned}
& M=\left\langle s_{0}\right\rangle(t)+[\tanh (2 K)]\left[S_{0}(t)-\bar{S}_{0}\right], \\
& \quad(t \rightarrow \infty, \text { rapidly varying boundary conditions }) .
\end{aligned}
$$




\subsection{Semi-infinite lattice with two parts of uniform cou- plings: the particular solution corresponding to har- monic boundary conditions}

Consider a semi-infinite lattice consisting of two parts, so that

$$
K_{\alpha}=\left\{\begin{array}{ll}
K_{1}, & \alpha<N \\
K_{2}, & \alpha>N
\end{array},\right.
$$

The time evolution equations for the expectation values of the spins are

$$
\begin{array}{rlrl}
\left\langle\dot{s}_{j}\right\rangle & =-2\left\langle s_{j}\right\rangle+\left[\tanh \left(2 K_{1}\right)\right]\left(\left\langle s_{j-1}\right\rangle+\left\langle s_{j+1}\right\rangle\right), & & 0<j<N, \\
\left\langle\dot{s}_{N}\right\rangle & =-2\left\langle s_{N}\right\rangle+\kappa_{-}\left\langle s_{N-1}\right\rangle+\kappa_{+}\left\langle s_{N+1}\right\rangle, \\
\left\langle\dot{s}_{j}\right\rangle & =-2\left\langle s_{j}\right\rangle+\left[\tanh \left(2 K_{2}\right)\right]\left(\left\langle s_{j-1}\right\rangle+\left\langle s_{j+1}\right\rangle\right), \quad N<j .
\end{array}
$$

where

$$
\begin{aligned}
& \kappa_{-}:=\tanh \left(K_{1}+K_{2}\right)+\tanh \left(K_{1}-K_{2}\right), \\
& \kappa_{+}:=\tanh \left(K_{1}+K_{2}\right)-\tanh \left(K_{1}-K_{2}\right) .
\end{aligned}
$$

Applying a harmonic boundary condition (23), one has for the particular solution of the kind (24),

$$
\sigma_{j}= \begin{cases}\left(A_{1} z_{1}^{j}+B_{1} z_{1}^{-j}\right), & 0 \leq j \leq N \\ A_{2} z_{2}^{j}, & N \leq j\end{cases}
$$

where

$$
z_{l}+z_{l}^{-1}=\frac{-\mathrm{i} \omega+2}{\tanh \left(2 K_{l}\right)}, \quad l=1,2
$$

and $\left|z_{l}\right|$ is smaller than one. The boundary condition results in

$$
A_{1}+B_{1}=\sigma_{0} .
$$

From (57) for $j=N$, one arrives at

$$
A_{1} z_{1}^{N}+B_{1} z_{1}^{-N}=A_{2} z_{2}^{N} .
$$

Finally, (54) results in

$$
\kappa_{-}\left(A_{1} z_{1}^{N-1}+B_{1} z_{1}^{-N+1}\right)+\kappa_{+} A_{2} z_{2}^{N+1}=(-\mathrm{i} \omega+2) A_{2} z_{2}^{N} .
$$

Equations (59) through (61) give

$$
\begin{aligned}
A_{1} & =\frac{\left(\kappa_{-} z_{1}+\kappa_{+} z_{2}+\mathrm{i} \omega-2\right) z_{1}^{-N} \sigma_{0}}{\kappa_{-}\left(z_{1}^{-N+1}-z_{1}^{N-1}\right)+\left(\kappa_{+} z_{2}+\mathrm{i} \omega-2\right)\left(z_{1}^{-N}-z_{1}^{N}\right)}, \\
B_{1} & =\frac{-\left(\kappa_{-} z_{1}^{-1}+\kappa_{+} z_{2}+\mathrm{i} \omega-2\right) z_{1}^{N} \sigma_{0}}{\kappa_{-}\left(z_{1}^{-N+1}-z_{1}^{N-1}\right)+\left(\kappa_{+} z_{2}+\mathrm{i} \omega-2\right)\left(z_{1}^{-N}-z_{1}^{N}\right)}, \\
A_{2} & =\frac{\kappa_{-}\left(z_{1}-z_{1}^{-1}\right) z_{2}^{-N} \sigma_{0}}{\kappa_{-}\left(z_{1}^{-N+1}-z_{1}^{N-1}\right)+\left(\kappa_{+} z_{2}+\mathrm{i} \omega-2\right)\left(z_{1}^{-N}-z_{1}^{N}\right)} .
\end{aligned}
$$


For large $N$, these simplify to

$$
\begin{aligned}
& A_{1}=\sigma_{0}, \\
& B_{1}=\frac{-\left(\kappa_{-} z_{1}^{-1}+\kappa_{+} z_{2}+\mathrm{i} \omega-2\right) z_{1}^{2 N} \sigma_{0}}{\kappa_{-} z_{1}+\kappa_{+} z_{2}+\mathrm{i} \omega-2}, \\
& A_{2}=\frac{\kappa_{-}\left(z_{1}-z_{1}^{-1}\right) z_{2}^{-N} z_{1}^{N} \sigma_{0}}{\kappa_{-} z_{1}+\kappa_{+} z_{2}+\mathrm{i} \omega-2} .
\end{aligned}
$$

It can be easily shown that for the nonuniform lattice and at high frequencies, up to first term in $\omega^{-1}$ the magnetization is similar to the case of the uniform lattice.

\subsection{Semi-infinite lattice with nonuniform couplings: the relaxation times}

The general solution of (9) with (12) and (13) is the sum of a particular solution and the general solution to (9) and (12) and (13) with vanishing $f$. The latter (the homogeneous solution) satisfies

$$
\begin{aligned}
\frac{\mathrm{d}}{\mathrm{d} t}\left\langle s_{j}\right\rangle_{\mathrm{h}}= & -2\left\langle s_{j}\right\rangle_{\mathrm{h}}+\left[\tanh \left(K_{j-\mu}+K_{j+\mu}\right)+\tanh \left(K_{j-\mu}-K_{j+\mu}\right)\right]\left\langle s_{j-1}\right\rangle_{\mathrm{h}} \\
& +\left[\tanh \left(K_{j-\mu}+K_{j+\mu}\right)-\tanh \left(K_{j-\mu}-K_{j+\mu}\right)\right]\left\langle s_{j+1}\right\rangle_{\mathrm{h}}, \quad 1 \leq j \leq L, \\
\left\langle s_{0}\right\rangle_{\mathrm{h}}= & 0, \\
\left\langle s_{L+1}\right\rangle_{\mathrm{h}}= & 0,
\end{aligned}
$$

which can be written as (17). Denoting an eigenvalue of $h$ by $E$, and the corresponding eigenvector by $\psi_{E}$, it is seen that there are solutions to (64) of the form

$$
\left\langle s_{j}\right\rangle_{\mathrm{h}}(t)=\psi_{E j} \exp (E t)
$$

These solutions decay with a relaxation time $\tau$ satisfying

$$
\tau=-\frac{1}{\operatorname{Re}(E)}
$$

One can see that the eigenvalues of the operator $h$ are real. To see this, one notices that equations (64) are the same as the equations corresponding to the homogeneous solution of (8). So the homogeneous solution to the Ising chain externally driven at ends, is the same as the homogenous solution to the Ising chain with magnetic fields at boundaries. The evolution equation for the latter satisfies the detailed balance. For any evolution satisfying detailed balance, the eigenvalues of the evolution operator are real. To see this, one notices that the criterion of the detailed balance is

$$
\omega(B \rightarrow A)=Y_{B}^{A} \exp \left[\beta\left(\mathcal{E}_{B}-\mathcal{E}_{A}\right)\right],
$$

where $A$ and $B$ are two different state, $Y_{B}^{A}$ 's are real nonnegative numbers (for $B \neq A$ ) satisfying

$$
Y_{A}^{B}=Y_{B}^{A},
$$


and $\mathcal{E}$ is the energy of the system in the state $A$. So the matrix $Y$ is Hermitian. Equation (67) means that the evolution matrix $H$, the components of which are $\omega(B \rightarrow A)$ 's, is a similarity-transformed of $Y$. As $Y$ is Hermitian, the eigenvalues of $Y$ are real. As $H$ is a similarity-transformed of $Y$, the eigenvalues of $H$ are the same as the eigenvalues of $Y$. So the eigenvalues of $H$ are real ([16 for example). The eigenvalues of $h$ are eigenvalues of $H$ as well. So the eigenvalues of $h$ are real.

\section{Concluding remarks}

A one dimensional kinetic Ising model at temperature $T$, with time varying boundary conditions was studied, for the case the lattice is semi-infinite. The evolution equation for the expectation values of the spins was investigated. For the case of harmonic boundary conditions, with uniform couplings, exact particular solutions were obtained for the expectation values of the spins, as well as the total magnetization. The low- and high-frequency behaviors were studied in more detail. Models for which the coupling constant is nonuniform were also studied. Physically, such nonuniform couplings could arise when either the interaction between spins or the temperature depends on the position. As a specific example, the harmonic solution on a semi-infinite lattice consisting of two homogeneous parts studied. Finally, it was shown that for a general (nonuniform) lattice, the eigenvalues corresponding to the evolution operator are real.

Acknowledgement: This work was supported by the research council of the Alzahra University. 


\section{References}

[1] R. J. Glauber; J. Math. Phys. 4 (1963) 294.

[2] M. Droz, J. Kamphorst Leal Da Silva \& A. Malaspinas; Phys. Lett. A115 (1986) 448

[3] T. Vojta; Phys. Rev E55 (1997) 5157.

[4] R. B. Stinchcombe, J. E. Santos, \& M. D. Grynberg; J. Phys. A31 (1998) 541.

[5] C. Godrèche \& J. M. Luck; J. Phys. A33 (2000) 1151.

[6] M. Droz, Z. Rácz, \& J. Schmidt; Phys. Rev. A39 (1989) 2141.

[7] C. Godrèche; J. Stat. Mech. (2011) P04005.

[8] C. Chatelain; J. Phys. A A36 (2003) 1073.

[9] M. Khorrami \& A. Aghamohammadi, 2002 Phys. Rev. E65 056129.

[10] M. Khorrami \& A. Aghamohammadi, 2008 arXiv:0811.2283.

[11] A. Aghamohammadi \& M. Khorrami; J. Stat. Mech. (2010) P1001.

[12] V. Popkov, M. Salerno \& G. M. Schutz; Phys. Rev E78 (2008) 011122.

[13] G. M. Buendi \& P. A. Rikvol; Phys. Rev. E78 (2008) 051108.

[14] A. Prados, L. L. Bonilla \& A. Carpio; J. Stat. Mech. (2010) P06016.

[15] L. L. Bonilla, A. Prados, \& A. Carpio; J. Stat. Mech. (2010) P09019.

[16] Geoffrey R. Grimmett \& David R. Stirzaker; "Probability and random processes" (Oxford University Press, 2001) third edition, section 6.14. 